\title{
Nursing care in palliative care in pediatric oncology
}

\begin{abstract}
This research has as general theme "Nursing care in palliative care in pediatric oncology", an approach that needs to be further discussed due to the need to prepare nursing professionals working in the pediatric sector with cancer patients who are terminally. Thus, an informative text was produced with information based on authors who have already conducted research on this theme, with a qualitative approach, because this is a Bibliographic research. To guide this research, the general objective was to identify the knowledge and reactions of nursing professionals in pediatric cancer care and as specific objectives to identify the perceptions and feelings that permeate pediatric nursing practice in relation to cancer, treatment, and its implications and to know the care/care activities aimed at children hospitalized in the pediatric oncology sector. It is concluded that the courses directed to nursing need to offer disciplines that prepare these professionals to deal with pediatric patients who are in terminal state, preparing them to comfort the family in the face of the failure of the treatments that the patient underwent.
\end{abstract}

Keywords: nursing, palliative care, pediatric oncology
Volume 5 Issue 3 - 202I

\author{
Joana Muniz Mendonça,' Luana Luzia de \\ Assis Arruda Corbari, ${ }^{2}$ Matheus Mychael \\ Mazzaro Conchy, ${ }^{3}$ Renan da Silva Bentes ${ }^{4}$ \\ 'Specialist Nurse in Oncology, Pediatrics and Neonatology, State \\ University of Roraima, Rua 7 de Setembro, 23I, Canarinho, Boa \\ Vista - RR, Brazil \\ ${ }^{2}$ Specialist Nurse in Pediatric Intensivism and Neonatology, \\ Roraimense College of Higher Education, Av. Presidente \\ Juscelino Kubitscheck, 300 Bairro Canarinho, Boa Vista - RR, \\ Brazil \\ ${ }^{3}$ Medical student at the Federal University of Roraima, Av. Cap. \\ Ene Garcês, ${ }^{\circ} 2413$ - Aeroporto, Boa Vista - RR, Brazil \\ ${ }^{4}$ Medical student at the Federal University of Roraima, Av. Cap. \\ Ene Garcês, n ${ }^{\circ} 2413$ - Aeroporto, Boa Vista - RR, Brazil
}

Correspondence: Matheus Mychael Mazzaro Conchy, Medical student at the Federal University of Roraima,Av. Cap. Ene Garcês, n 2413 - Aeroporto, Boa Vista - RR, Brazil, Email matheusmazzaro03@gmail.com

Received: May 18, 2021 | Published: June 09, 2021

\section{Introduction}

Pediatric cancer patients generally arouse greater empathy in people than adults. The team of health professionals sympathizes with the pain and suffering of the patient's family since it is a painlike and distressing treatment due to expectations with waiting for the positive result of the treatments they undergo. In view of this approach, it is the nursing professionals who are at the front of the care and performance of the procedures of cancer patients, so they need to be prepared to attend to families and patients, because this is a very delicate time for both. Therefore, it is essential that academic courses provide disciplines that prepare these professionals physically and psychologically to meet this demand of patients. Therefore, this is a bibliographical research to meet the proposed objectives because the general objective was to identify the knowledge and reactions of nursing professionals in pediatric cancer care and as specific objectives to identify the perceptions and feelings that permeate pediatric nursing practice in relation to cancer, treatment, and its implications and to know the care/care activities aimed at children hospitalized in the pediatric oncology sector. This work is structured with the theoretical foundation where it will be addressed on nursing care in palliative care in pediatric oncology, divided into two topics, pediatric oncology and palliative care in pediatric oncology performed by nursing professionals.

\section{Nursing care in palliative care in pediatric oncology \\ Pediatric oncology}

Cancer treatment has become the great villain of medicine, as there are numerous researches aimed at finding a cure, however research has not yet found a treatment that allows the cure of all types of cancers, causing frustration for researchers and patients. Currently, cancer affects millions of people worldwide, and for some types of cancer the chances of cure are higher than in others. In addition, the chances of cure are considerably higher when the cancer is discovered in the early stage. When addressing cancer in children, amore cautious approach is needed, due to the numerous procedures that the child needs to undergo. For, childhood and juvenile cancer is a problem that affects children from 0 to 19 years, corresponding to $1 \%$ to $3 \%$ of malignant tumors in most populations. In Brazil, there is a very significant number of cancer in children. In this sense, Guimaraes et a. ${ }^{1}$ states that:

[...] approximately 9,386 cases of pediatric tumors are estimated to appear per year. Survival has increased over the years, currently reaching a rate of $70 \%$. This is due to factors such as early diagnosis and access to appropriate treatments.

Early diagnosis is still the best alternative for treatment, also facilitating the best choice of treatment for cure, since the original tumor can spread throughout the body, reach in go the organs, that is, causing metastases, thus reducing the chances of recovery of the patient. In this sense, the authors Guimarães et al. ${ }^{2}$ define the "Cancer is a set of diseases that have in common the uncontrolled proliferation of abnormal cells, which invade tissues and organs, and can spread to various parts of the body, causing metastasis." Therefore, it is important that the diagnosis occurs early so that the patient is more likely to recover with the necessary treatment for the type of cancer he faces.

Children facing cancer problems need to receive specialized treatments with humanized follow-up by health professionals who are part of the team that offer "preventive, curative and palliative" care. In this sense, the authors Mutti et al. ${ }^{3}$ explain how the type of preventive treatment occurs:

Preventive care in the field of oncologic pediatrics can be developed by actions before the birth of the child and during childhood. Before birth, genetic counseling to parents has been shown to be a possibility in prevention. During childhood, with guidance on healthy living habits, such as: eating, physical activity and care for the 
environment. These prevention cares are fundamental for the health of the baby when born, providing health for children, and preventing against future health problems, or emergence of cancer, since there is an exceptionally large number of children who have acquired cancer generated from numerous health problems. In the treatment and curative care, the nursing professional acts directly in all phases, diagnosis, treatment, and control. Due to this, the authors Neves Mendes et al. ${ }^{4}$ contribute:

Curative care involves the phases of diagnosis, treatment, and control. The nursing professional should be attenuated in all these phases, providing care in an integral way and seeking means to deal with the impact of diagnosis and treatment, offering the child and family all necessary support. Still as part of the curative care, there is the control phase, which happens after the end of cancer treatment, and there may or may not be recurrence of the disease.

These curative cares provide better results in cancer treatment, because cancer is a globally recognized health problem, because worldwide the number of cancers in children represents $0.5 \%$ to $3 \%$ when compared to the general population, and $1 \%$ to $4.6 \%$ in children in Brazil based on the Brazilian population. ${ }^{3}$

This is an incredibly significant number in the general population, as children facing health problems due to cancer vary in age and influencing factors.

Palliative care in pediatric oncology is a more significant approach because palliative care is directed to children with cancer who have no cure. Yes, itis care that is directed to give more comfort to the patient who is without life expectancy. In this context, Guimaraes et al. ${ }^{1}$ states "Palliative care in pediatric oncology involves several aspects, such as the impossibility of cure, the breakdown of life expectancy that is expected for the child, the end of a fragile being that is protected in our culture and family." Therefore, it is important to highlight that during the treatment period, pediatric oncology patients need to receive a humanized treatment from health professionals who are part of the team that attends the Neonatal Intensive Care Unit.

\section{Palliative care in pediatric oncology performed by nurses}

Palliative care for oncology patients is fundamental for the comfort of the patient and the family, as this is a time of great tension for all who are part of this hospitalization process, dis structuring families, disorganizing their routine, and providing moments of pain and sadness for all. In view of this context, the authors Paro Paro and Ferreira ${ }^{5}$ highlight:

Given the diagnosis of a disease such as cancer, the family usually disorganizes, changing routine and dynamics, thus requiring accompanied and assisted inclusion. Poor communication between parents and health staff and between parents and children results in serious consequences for the child's health.

The dialogue between the family and the health team is important for the patient's health, which needs to be guided on how all procedures will occur, what recommendations should be followed in the follow-up, in medications, basic health care about each stage of the disease. It is known that there are different forms of treatment for cancer, which can be used together or in isolation, but it is important that the healthcare team that attends and pediatric oncology patients be humanized to explain the risks related to the disease and treatment, the consequences that can aggravate the patient's healthstatus. When families resort to these treatments, they expect positive results. But when this does not happen families experience moments of frustration and suffering. In this sense, Guimaraes et al. ${ }^{1}$ explains better how this happens:

Several treatments are used to treat and cure chronic diseases, including cancer in children. Technological advances, powerful medications, new diagnostic techniques are employed in an attempt to succeed in curing the patient, reinforcing care based on interventionism and curative. However, often success does not occur, and the disease does not respond to treatment, exhausting the possibilities of cure, which leads the team to adopt a new approach of care for that patient: palliative care.

This is the worst part between diagnosis and treatment, since the discovery of cancer is already a moment of shock, but when they discover that he has no cure and that the treatment alternatives offered did not get the expected result, the family find themselves facing an unexpected situation, pain, and sadness.

At this time, parents are the spokespersons of the child, so it is essential, "[...] represent the feelings, attitudes, sociocultural behaviors internalized in the world of the child's life. They are the mediators of the child in the world of the hospital. ${ }^{6}$

As for palliative care, it is necessary that the professional responsible for care is very patient and has skills to facilitate communication between family and health professionals. Therefore, the World Health Organization establishes what palliative care is. And in this sense the authors Guimaraes et al. ${ }^{2}$ explains:

The World Health Organization (WHO) defines palliative care "as an approach aimed at improving the quality of life of patients and their families, who face problems associated with life-threatening diseases. Carried out through the prevention and relief of suffering, by early identification, correct assessment and treatment of pain and other physical, psychosocial and spiritual problems".

The professional to perform this function, needs to have in addition to an academic training, he must provide humanized care. That is why the need for humanization in health. Among the outstanding professionals, we have the nurse, a professional who develops many functions within the health services that serve cancer patients.

According to the authors Vieira, Castro and Coutinho" "The nursing care provided to these small patients generally takes care of a series of techniques related to hygiene, feeding, collection of material for examinations and administration of medication." Therefore, it is important that these professionals take courses that prepare you for this type of work.

In this context, the authors Neves, Mendes and Santos (s. d. p. 09) point out that:

Humanization requires nursing professional's knowledge, disposition, active interest, affectivity, flexibility, search to improve care, responsibility, sensitivity, ability to listen to the expression of feelings without pre-judgments or censorship.

And when it comes to cancer in pediatrics, it is a disease that imposes changes in the life of the family, causing suffering and expectations for the family and the child, because it modifies their lives, especially their routine. And the authors Paro Paro and Ferreira ${ }^{5}$ highlight that "Social, emotional, affective, cultural and spiritual aspects form a context that subject patients and their families to phases that do not necessarily result from the evolution of pathology."

However, all these problems are the result of a lot of pressure suffered by the family and the patient when he is already a child who 
understands what is happening to his health. Because cancer in children usually arouses more intense empathy for health professionals. "For younger children, cancer may be related to punishments for misconduct. The deprivation of the cervix, the coziness of the parents in the intervention procedures, cause great stresses for children" (PARO, PARO and FERREIRA, 2005, p. 152)".

Thus, it is important that nurses are prepared to care for patients and families in palliative care, in this sense the authors Paro Paro and Ferreira $^{5}$ also emphasize:

When caring for the child, one should understand his/her world and the stages of childhood, holistically about the child-family dyad, seeking to satisfy his/her needs, regardless of his/her current condition. The nursing team, together with the interdisciplinary team, should develop activities with the child and his/her family, seeking to maintain well-being. Based on these principles, we will turn our attention to nurses, professionals who may be present during the intervention and treatment processes, interacting in the care of cancer patients.

Therefore, it is noted that it is necessary that the nursing professional is professionally and psychologically qualified to support the families and patients, since this is a difficult time for her. However, the reality is quite different from what is expected because some studies published in the literature directed at health, states that nurses feel unprepared to face this situation, give bad news to families, deal with patients and face lossand live the mourning. ${ }^{1}$

Currently, nurses question the lack of training to deal with families, physical exhaustion, and emotion al, lack of continuing education and lack of inclusion of this theme in professional training courses, because nurses are trained to deal with patients, but not prepared to deal with the death of pediatric oncology patients.

Therefore, it is important that nursing training courses approach with students about palliative care in pediatric oncology, in addition to sensitizing them to their professional future. And the authors Mutti Paula and Souto ${ }^{10}$ reaffirm, "It is perceived that the specialty of pediatric oncology is contemporary and has its body of knowledge and practice in the process of construction."

For the authors Silva Issi and Motta ${ }^{7}$ state:

Therefore, the main objective of palliative care is to add quality of life to days, not days to life. This represents a great challenge for the nursing team, since, with the appreciation of care, these are the professionals who most experience the reality of the patient, and they have the responsibility to rescue the self-esteem, comfort and individuality of the patient and his/her family.

Palliative care is fundamental in this process, because when pediatric cancer is diagnosed early, there is a $70 \%$ chance of cure, but when they are treated in specialized centers. But when all types of curative treatment are exhausted, such as surgery, radiotherapy, chemotherapy, and/or transplantation, only palliative care remains, which is the treatment directed to the comfort of families and the child. $^{2}$

The expectation of overcoming a cancer is a time long awaited by the family, and when this does not occur as expected, it becomes frustrating for everyone who is involved. In this sense, the authors Neves, Mendes and Santos (s. d, p. 05 and 06) contribute to these perspectives, saying:

Experiencing cancer is more painful for customers and their families than any other disease. For, in a singular way, the neoplasm arouses several negative feelings in any of its stages: the shock of the diagnosis, the fear of surgery, the uncertainty of prognosis and recurrence, the effects of chemo and radiotherapy, fear and the possibility of death.

For these reasons, it is necessary that the patient and the family receive a humanized treatment currently, because Neves, Mendes and Santos (s. d, p. 06) state that it is "Amore humane care, which respects their condition as a child, reduces stress in relation to the disease and therapy, which also has consequences in the way of how nursing mothers face treatment, along with their child." This treatment directly influences the patients psychological.

Given this approach, it is essential that nursing students be prepared for these situations that they will experience in their workplace, especially how they should approach the family with the bad news and with the procedures they will perform with the patient. In this context, Neves, Mendes and Santos (s. d. p. 06) still infer that:

The child has limited ways of facing adverse situations and, in the case of hospitalization, institutions need to act to promote more familiar and humanized and less threatening environments. The offer of means for children to play makes it possible to cope with the adverse effects of cancer. Adapting the hospital environment to children's needs makes the delicate situation faced by the child become milder.

The child is always willing to participate in everything he/she experiences, and when it comes to hospitalization is an adverse situation that no one is prepared to experience, given this, it is important that the professional who is accompanying him, is humanized, because the communication between then nurse and family needs to be enlightening, that makes the child understand what he is facing, because it is known that he will not understand all the procedures, but will allow him to experience in his day-to-day everything that is happening clearly.

\section{Conclusion}

Curative care and palliative care are fundamental in the treatment of cancer patients, and when it comes to cancer in children is even more important, because curative care provides moments of hope for the patient and family who are waiting for a cure with the regression of the disease with the treatments performed. However, this does not always occur, because in some cases cancer advances and has no cure, so palliative care in pediatric oncology must take place, because it is a time of great stress, fear, anguish and suffering for families and patients, with this it is important that the health professional who is accompanying the case offers humanized treatment.

The role of nurses in this stage is essential because the nurse accompanies the pediatric oncology patient in all procedures, in view of this, he performs palliative care, offering comfort, explaining what is happening, providing the patient and the family with a more dignified treatment, since it is a delicate moment for both. Therefore, it is concluded that the courses directed to nursing need to offer disciplines that prepare these professionals to deal with pediatric patients who are in terminal condition, preparing them to comfort the family in the face of the failure of the treatments that the patient underwent.

\section{Acknowledgments}

None.

\section{Conflicts of interest}

The author declares there is no conflict of interest. 


\section{References}

1. Guimarães, Tuani Magalhães, Fátima Helena Espírito Santo, et al. Palliative care in pediatric oncology in nursing education. Rev Gaucho Enferm. 2017;38(1):e65409.

2. Guimarães, Tuani Magalhães, Fátima Helena Espírito Santo, et al. Palliative care in pediatric oncology in the perception of nursing students. Anna Nery School. 2016;20(2).

3. Mutti, Cintia Flores, Paula. Health Care for Children with Cancer in Brazilian Scientific Production. Brazilian Journal of Cancerology. 2010;56(1):71-83.

4. Marques Rosângela, Cordeiro Jeandro Oliveira, Mugnol Katia Cristina Ugolini. Coping with nursing professionals in palliative care in Pediatric Oncology: A reflexive analysis of emotions. S.D. 2020
5. Paro Daniela, Paro Juliana, Ferreira Daise LM. Nurse and care in Pediatric Oncology. Therq Ciênc Saúde, 2005;12(3):151-157.

6. Silva, Adriana Ferreira da ISSI, Helena Becker MOTTA. The family of the oncologic child in palliative care: the look of the nursing team. Cienc Cuid Saúde. 2011;10(4):820-827.

7. Neves Jessica Nunes, Mendes Daniella RG, Santos Walquíria Lene dos. Pediatric Oncology Nursing: Factors of excellence in comprehensive care. S.D. 2020

8. Vieira Amanda Patez, Castro Daniele Lima, Coutinho Mislene Silva Nursing care in pediatric oncology. Rev Eletrôn Updates Health | Salvador. 2016;3(3):67-75. 